dose of MTX ( $\geq 15 \mathrm{mg} / \mathrm{wk}$ ) (HM-group). A multinomial logistic regression model was used to test the association between MTX start dose and EULAR response at 6 months,with adjustments (see table) (relative risk ratio (RRR), 95\% Cl). The model was clustered by centre to account for the prescribing preferences of individual centres.

Results: 810 patients were included in this study: 171/810 (21\%) starting low dose MTX and 639/810 (79\%) starting high dose MTX. Patients in the HM-group had significantly lower physician and patient VAS scores and less functional disability compared to those in the LM-group (table). These patients were also less likely to be prescribed concomitant (nbDMARDs) (17\% vs. 10\%). DAS28 score at 6 months was significantly lower for patients in the HM-group.

A fully adjusted multinomial logistic regression model, clustered by centre, showed that being in the high dose MTX group does not affect the odds of having a moderate response rather than no response, but does increase the odds of having a good response (RRR: 2.65 (95\% Cl 1.37, 5.14)).

Conclusions: Patients with RA starting MTX on a higher dose have increased odds of having a good EULAR response compared to non-response at 6 months. References:

[1] Visser, K. et al (2009). Multinational evidence-based recommendations for the use of methotrexate in rheumatic disorders with a focus on rheumatoid arthritis: integrating systematic literature research and expert opinion of a broad international panel of rheumatologists in the $3 E$ Initiative. Ann Rheum Dis, 68: 1086-1093.

Disclosure of Interest: None declared

DOI: 10.1136/annrheumdis-2017-eular.6047

\section{OP0226 SUSTAINED EFFECTIVENESS OF METHOTREXATE WITH STEP-DOWN GLUCOCORTICOID REMISSION INDUCTION (COBRA SLIM) FOR EARLY RHEUMATOID ARTHRITIS IN A TREAT-TO- TARGET SETTING: 2-YEAR RESULTS OF THE CARERA TRIAL}

V. Stouten ${ }^{1}$, J. Joly ${ }^{2}$, D. De Cock $^{1}$, S. Pazmino ${ }^{1}$, K. Van der Elst ${ }^{2,3}$ R. Westhovens ${ }^{1,2}$, P. Verschueren ${ }^{1,2}$ on behalf of CareRA study group. ${ }^{1}$ Skeletal Biology and Engineering Research Center, KU Leuven Department of Development and Regeneration; ${ }^{2}$ Rheumatology, University Hospitals Leuven; ${ }^{3}$ Skeletal Biology and Engineering Research Center, KU Leuven Department of Public Health and Primary Care, Leuven, Belgium

Background: The CareRA trial showed that remission induction with MTX and a moderate-dose of Glucocorticoids (GC) (COBRA Slim) in a treat-to-target setting is effective and safe in early Rheumatoid Arthritis (eRA) patients. This strategy showed equally high remission rates at 52 weeks (W), a favourable safety profile compared to DMARD combinations and GC and very few patients had to start biologicals.

Objectives: To compare the outcome of different intensive combination treatment strategies in high-risk patients of the CareRA trial at W104, focussing on persistent disease control.

Methods: CareRA is a two-year prospective investigator-initiated pragmatic multicentre RCT; csDMARD naïve eRA patients were stratified into a high- or low-risk group based on classical prognostic markers (presence of erosions, $R F$, anti-CCP and DAS28-CRP). High-risk patients $(n=289)$ were randomized to $1 / 3$ arms: 1) COBRA Classic $(n=98)$ : Methotrexate (MTX)+Sulphasalazine+60mg prednisone tapered weekly to $7.5 \mathrm{mg}$ daily from W7; 2) COBRA Slim $(n=98)$ : MTX+30mg prednisone tapered to $5 \mathrm{mg}$ daily from W6; 3) COBRA Avant-Garde $(\mathrm{n}=93)$ : MTX+Leflunomide+30 mg prednisone tapered to $5 \mathrm{mg}$ daily from W6. From W28, GCs were tapered in all patients and stopped at W34. A predefined treat-to-target approach was applied until W52 and afterwards treatment was at the discretion of the rheumatologist. From W40, DMARD monotherapy was aimed for. From W28 onwards patients were evaluated every 3 months till W104. Efficacy measures were proportions of DAS28-CRP remission, good EULAR response, clinically meaningful $\mathrm{HAQ}$ response, $\mathrm{HAQ}=0$ (ITT analysis). Adverse events related to therapy (AEs) were registered. Missing data were imputed by last observation carried forward.

Results: Remission rates at W104 in high-risk patients were $65.3 \%, 73.5 \%$ and $73.1 \%$ in the Classic, Slim and Avant-Garde group respectively $(p=0.369)$. Also, other efficacy outcomes did not differ between groups (see table). From the high-risk patients that were in remission at year 1, 54.7\%,67.8\% and $70.2 \%$ in the Classic, Slim and Avant-Garde group respectively, stayed in remission at

\begin{tabular}{|l|c|c|c|c|}
\cline { 2 - 5 } \multicolumn{1}{c|}{} & $\begin{array}{c}\text { COBRA } \\
\text { classic } \\
\mathrm{N}=98\end{array}$ & $\begin{array}{c}\text { COBRA slim } \\
\text { (high-risk) } \\
\mathrm{N}=98\end{array}$ & $\begin{array}{c}\text { COBRA } \\
\text { Avant-Garde } \\
\mathrm{N}=93\end{array}$ & p-value \\
\hline DAS28-CRP change BL-w104 & $2.6 \pm 1.4$ & $2.6 \pm 1.3$ & $2.6 \pm 1.6$ & 0.966 \\
\hline DAS28-CRP change w52-w104 & $0.0 \pm 1.0$ & $0.2 \pm 1.0$ & $0.3 \pm 1.1$ & 0.112 \\
\hline DAS28 Remission w52 & $65.3 \%$ & $60.2 \%$ & $61.3 \%$ & 0.741 \\
\hline DAS28 Remission w104 & $65.3 \%$ & $73.5 \%$ & $73.1 \%$ & 0.369 \\
\hline Good EULAR response & $78.6 \%$ & $81.6 \%$ & $78.5 \%$ & 0.826 \\
\hline Clinically meaningful HAQ change & $70.4 \%$ & $62.2 \%$ & $70.7 \%$ & 0.363 \\
\hline HAQ =0 & $39.8 \%$ & $37.8 \%$ & $38.7 \%$ & 0.958 \\
\hline
\end{tabular}

DAS28-CRP $=28$ Joint disease activity score calculated with C-reactive protein; remission = DAS28-CRP $<2.6$; low disease activity $=$ DAS-CRP $\leq 3.2$; good EULAR response $=$ low disease activity with a DAS28 CRP change $>1.2 ; \mathrm{HAQ}=$ health assessment questionnaire; clinically meaningful $\mathrm{HAQ}$ change $=\mathrm{HAQ}$ change $>0.22$. every three-monthly evaluation until w104. Also DAS28-CRP scores remained relatively stable during the second year in these groups. In high-risk patients, the total numbers of AEs reported as related to study therapy, were 209 in 72 Classic patients, 164 in 69 Slim patients and 208 in 74 Avant-Garde patients $(p=0.029)$. Serious AEs were reported in 3 Classic, 4 Slim and 3 Avant-Garde patients. Biologicals were started in 44 high-risk patients $(15.2 \%)$, of which 7 receiving 2 different biologicals and 2 receiving 3 different ones. Biologicals were administered in 18 Classic, 11 Slim and 15 Avant-Garde patients.

Conclusions: All groups showed persistently high remission rates 2 years after remission induction with csDMARDs and GCs in a treat to target setting. COBRA Slim showed comparable efficacy with less adverse events compared to DMARD combinations with moderate or high GC induction dosages. In almost $70 \%$ of COBRA Slim patients achieving remission at year 1, this was maintained throughout the second year.

Disclosure of Interest: None declared

DOI: 10.1136/annrheumdis-2017-eular.6472

\section{OP0227 REMISSION INDUCTION WITH METHOTREXATE STEP-UP THERAPY VERSUS COMBINATION OF HYDROXYCHLOROQUINE, METHOTREXATE AND TRIAMCINOLONE: 3 YEAR RESULTS}

T. Brunekreef, H. Bernelot Moens. Rheumatology \& Clinical Immunology, Ziekenhuisgroep Twente, Almelo, Netherlands

Background: The effectiveness of treat to target (T2T) in RA is widely acccepted, but there is no consensus regarding the best initial treatment in early rheumatoid arthritis ${ }^{1}$. Therefore, it is important to evaluate the results of such strategies in real life cohorts ${ }^{2,3}$

Objectives: Compare the effectiveness of step-up methotrexate (MTX) monotherapy and combination of hydroxychloroquine (HCQ), methotrexate and intramuscular injection of triamcinolone $80-120 \mathrm{mg}$ as initial treatment in early RA.

Methods: Historical cohorts of patients treated with MTX monotherapy (disease onset 2006-2011, N=297) and combination therapy (2012-2016, N=156) were compared. In both cohorts a b-DMARD was advised when no remission was reached within 6 months or in case of sustained activity thereafter. Baseline characteristics and disease activity (DAS28) measurements ( $N=4956$, average 4.1/year) in the first 3 years of follow-up were available. The primary outcome measure was the proportion of patients having reached at least one DAS28 $<2.6$ (remission) during follow-up. Secondary outcomes were sustained remission over 36 months and time to first b-DMARD.

Results: Three patients did not start MTX, and 11 and 2 patients in the step-up and combination cohorts respectively did not have complete follow-up (Table). Within 12 months, more patients on combination treatment reached remission $(88,2 \%$ vs $72.2 \%)$. In the second year these changed to $86.5 \%$ and $82.0 \%$ respectively. Combination treatment resulted in a higher percentage of DAS measurements below 2.6 over 3 years, reflecting sustained remission (Figure). A b-DMARD was started within 24 months in $20.6 \%$ of patients on monotherapy versus $14.1 \%$ on combination treatment, with an equal mean time to first b-DMARD of 12 vs 11 months after start of initial treatment.

\begin{tabular}{lcc} 
Table 1 & \\
\hline Table & MTX monotherapy (N=297) & MTX with HCQ and steroids (N=156) \\
\hline Female, N (\%) & $180(60.6)$ & $101(64.7)$ \\
Age (y), mean (SD, range) & $59.5(14.3,18-89)$ & $112(71.8)$ \\
Rheumatoid factor (N, \%) & $183(61.6)$ & $111(71.2)$ \\
ACPA (N, \%) & $157(65.3)$ & $42(11-62)$ \\
Follow-up (months) (range) & $81(8-132)$ & \\
End of follow-up <3 yrs & & $1(0.6 \%)$ \\
$\quad$ Death & $4(1.3 \%)$ & $1(0.6 \%)$ \\
$\quad$ Remission & $4(1.3 \%)$ & $1(0.6 \%)$ \\
$\quad$ Did not start MTX/Other & $5(1.7 \%)$ & \\
Any remission (DAS28 <2.6) & & $88.2 \%$ \\
$\quad$ First year & $72.2 \%$ & $86.5 \%$ \\
Second year & $82.0 \%$ & $87.0 \%$ \\
$\quad$ Third year & $85.9 \%$ & \\
Start b-DMARD therapy & & $17(10.9 \%)$ \\
$\quad$ First year & $32(10.8 \%)$ & $5(3.2 \%)$ \\
Second year & $29(9.8 \%)$ & $5 / 104(4.8 \%)$ \\
$\quad$ Third year & $8(2.7 \%)$ & \\
\end{tabular}

Conclusions: Combination treatment results in more remissions in the first year of treatment. In the second and third year the remission percentage on monotherapy comes close to combination treatment, at the cost of a $6 \%$ higher proportion of patients stepping up to biologicals. Overall, the combination of MTX with $\mathrm{HCQ}$ and triamcinolone results in more sustained remissions.

References:

[1] Stoffer M, Schoels M, Smolen J et al. Evidence for treating rheumatoid arthritis to target: results from a systematic literature search update. Ann Rheum Dis 2016; 75:16-22.

[2] de Jong P, Hazes J, Barendregt P et al. Induction therapy with a combination of DMARDs is better than methotrexate monotherapy: first results of the tREACH trial. Ann Rheum Dis 2013; 72:72-8.

[3] Steunebrink L, Versteeg G, Vonkeman H et al. Initial combination therapy versus step-up therapy in treatment to the target of remission in daily clinical 